\title{
DISTRIBUTION OF LOW-POWER SOLAR FLARES BY BRIGHTNESS RISE TIME
}

\author{
A.V. Borovik \\ Institute of Solar-Terrestrial Physics SB RAS, \\ Irkutsk, Russia,aborovik@iszf.irk.ru
}

\author{
A.A. Zhdanov \\ Institute of Solar-Terrestrial Physics SB RAS, \\ Irkutsk, Russia, kick.out@mail.ru
}

\begin{abstract}
Using data from the international flare patrol for 1972-2010, we have formed an electronic database for more than 123 thousand solar flares. We determined the mean brightness rise time (flash phase) for flare area classes and importance. We show that the mean flash phase increased with increasing area class. For brightness classes this trend is less pronounced. We have found that flares with explosive phase and flares with one brilliant point have the shortest flash phases; two-ribbon flares and flares with several intensity maxima, the longest ones. We have separated 572 cases when the brightness rise time was more than $60 \mathrm{~min}$;
\end{abstract}

$80 \%$ of such ultra-long flares have a shorter brightness decay time (main phase). We have established that lowpower flares in terms of developmental features do not differ from large flares. Low-power solar flares, as well as large flares, can be followed by filament activation or disappearance, and can have an explosive phase and several intensity maxima. Two-ribbon flares, white-light flares, and flares covering sunspot umbra can also have low power.

Keywords: solar activity, solar flares.

\section{INTRODUCTION}

Time parameters of solar flares in the $\mathrm{H} \alpha$ line have been examined in detail during formation years of the international flare patrol in the comprehensive research into global geophysical processes under international programs of the International Geophysical Year (IGY). The results have shown that flares tend to develop in two stages: initial (flash phase) and main phase. During the flash phase, flare brightness rises for several tens of seconds or tens of minutes (in some cases, for one hour). In the second (main) phase, it gradually decreases and within about one hour becomes as low as the plage brightness (in some cases, this may continue for days). A detailed analysis of time parameters of flares can be found in [Smith G., Smith E., 1966; Švestka, 1976; Altyntsev et al., 1982].

The flash phase is the most important period of the primary energy release from solar flares. It is attended with hard $\mathrm{X}$-rays and gamma rays, microwave radio bursts, radiation in chromospheric lines, continuum, ultraviolet, and extreme ultraviolet [Fletcher et al., 2011]. The most advanced solar flare model (CSHKP) available, which combines different observational and theoretical concepts [Sturrock, 1966; Hirayama, 1974; Kopp, Pneuman, 1976], suggests that the onset of flares and the rapid release of the energy stored in a magnetic field occur in the corona due to magnetic reconnection. From a coronal source, the energy is transferred along magnetic flux tubes to the chromosphere, where it appears as flare ribbons and footpoints in hard X-rays.

This paper deals with the duration of flash phases of solar flares in the Ha line. Special attention is given to low-power flares with an area of less than 2 sq. deg., which constitute the majority (over $90 \%$ ) of all solar flares [Borovik, Zhdanov, 2017]. Some studies carried out over the past two decades are presented in [Temmer et al., 2001; Giersch, 2013; Potzi et al., 2014]. The results of earlier studies have been obtained from relatively small data samples, mainly using the first international classification of solar flares of 1956, and therefore need clarifying.

\section{DATABASE AND ITS ANALYSIS}

Observations in the optical wavelength range due to its critical importance for diagnostics and prediction of nonstationary processes on the Sun have been made by observatories of the world for many years. According to the modern international classification, $\mathrm{H} \alpha$ flares fall into five classes of area: $\mathrm{S}, 1,2,3$, and 4. Each area class, in turn, is divided into three brightness classes: $\mathrm{F}$ (faint), $\mathrm{N}$ (normal), and $\mathrm{B}$ (bright). Optical importance is assigned to a flare, having regard to these two parameters. Nowadays, the most comprehensive databases of solar flares in the Ha line (6563 $\AA$ ) are catalogs of Solar Geophysical Data (SGD) and Quarterly Bulletin on Solar Activity. According to the current international classification, SGD provides data from 1975 to 2010; the Quarterly Bulletin, from 1972 to 1989. To use the maximum possible amount of data for the statistical analysis, SGD catalogs have been supplemented with flare data from the Quarterly Bulletin for 1972-1975. Software-based methods have been used to correct errors, misprints and inaccuracies. Duplication of the same events has been eliminated. The cases when stations did not report on brightness and area classes, time of onset, maximum, and end of a flare, etc. have been taken into account. As a result, we have formed an electronic database for 1972-2010, which contains parameters of 123801 solar flares; 110778 of them are low power, 11280 belong to class 1 , and 1743 have class 2 or higher.

As noted by many authors [Abramenko et al., 1960; Warwick, 1965; Smith G., Smith E., 1966 Kopetskaya, Kopetsky, 1971; Ward et al., 1973; Rossada, 1977; Borovik, Zhdanov, 2017], international flare patrol data 
suffer from certain heterogeneity. In particular, the time of onset, maximum, and end of a flare may differ in group reports of different stations. This is due to the fact that the onset of a flare is usually determined with fairly high confidence. The time when the flare peaks or completely ends is difficult to determine: some areas fade quickly, others continue to "burn" for a long time. To estimate the variance of the rise time, we find standard deviations $\sigma$ in group reports from the stations:

$$
\sigma=\sqrt{\frac{1}{n-1} \sum_{i=1}^{n}\left(x_{i}-\bar{x}\right)^{2}} .
$$

First, from these groups we exclude summarized data obtained visually and under poor observation conditions as well as reports with letter qualifiers (D, E, U, and *). The obtained $\sigma$ values are then averaged over importance and area classes of flares. The mean values $(\bar{\sigma})$ are listed in Table 1 ( $N_{\mathrm{gr}}$ is the number of the analyzed groups).

Table 1

$\bar{\sigma}$ as a function of flare importance and area class

\begin{tabular}{|c|r|c|}
\hline & $N_{\text {gr }}$ & $\bar{\sigma}$ \\
\hline SF & 8433 & 2.2 \\
\hline SN & 2991 & 2.4 \\
\hline SB & 699 & 2.5 \\
\hline $1 \mathrm{~F}$ & 161 & 2.9 \\
\hline $1 \mathrm{~N}$ & 469 & 3.6 \\
\hline $1 \mathrm{~B}$ & 395 & 3.4 \\
\hline$(2-4) \mathrm{F}$ & 13 & 4.3 \\
\hline$(2-4) \mathrm{N}$ & 65 & 3.5 \\
\hline$(2-4) \mathrm{B}$ & 161 & 4.9 \\
\hline $\mathrm{S}$ & 16352 & 2.5 \\
\hline 1 & 1697 & 3.7 \\
\hline $2-4$ & 359 & 4.5 \\
\hline
\end{tabular}

The results suggest that as area class and importance increase $\bar{\sigma}$ gradually increases from 2.2 to $4.9 \mathrm{~min}$. To reduce the variance, large data samples or observations from a single station are usually utilized. In this work, we have implemented both the approaches.

\section{BRIGHTNESS RISE TIME ACCORDING TO DATA FROM ALL STATIONS}

For the statistical analysis, as in estimating the variance, from the database we have excluded the flares whose parameters are assessed visually, have poor observation conditions, and are marked with classifiers of uncertainty. Because of possible errors in identifying the flare importance near the solar limb, we omit class $\mathrm{S}$ and 1 solar flares that occur $65^{\circ}$ farther from the central meridian. For large flares (2-4), this restriction is not imposed. It is to be supposed that the area class for such flares should be easy to determine.

We abandon the use of summarized data in group reports. After applying all selection criteria, we select the station that has the greatest number of reports in the database. As a result of the selection, the total number of flares decreases to 84 628. For them, the distribution by brightness rise time (in percent of the total number of flares with increment of $1 \mathrm{~min}$ ) is illustrated in Figure 1. A vertical dashed line indicates the position of the median. The tail of the distribution is scaled up (axis at the right).

The distribution is rather asymmetric with a long tail. The rise time in some flares exceeds $240 \mathrm{~min}$ (not shown in the Figure). To assess more precisely the main statistical parameters of the distribution, its range is limited to $141 \mathrm{~min}$ - the time when the distribution continuity is disrupted. The unaccounted flares (27) amount to $0.03 \%$. Hereinafter, all calculations of the statistical parameters are made only within this range. To give more statistical significance to large flares, we combine class 2, 3, and 4 flares into group (2-4).

Figures 2-4 show distributions of brightness rise time for individual importance and area classes.

All the distributions exhibit a pronounced positive asymmetry - a pronounced maximum followed by an extended decrease. Additional peaks occurring in highimportance flares (Figure 4) are due most likely to a small amount of data. Table 2 lists statistical parameters of the distributions: number of flares $N$ of each importance and area class, mean brightness rise time $\bar{t}$ with a confidence interval $\alpha$, modal and median distribution parameters ( $\mathrm{Mo}, \mathrm{Me}$ ), time intervals $\Delta t$ for $90 \%$ of flares. All time parameters are given in minutes.

$$
\bar{t}-\Omega(P, n-1) \frac{\sigma}{\sqrt{n}} \leq \alpha \leq \bar{t}+\Omega(P, n-1) \frac{\sigma}{\sqrt{n}},
$$

where $\bar{t}$ is the mean rise time, $\Omega$ is Student's coefficient (1.96), $P$ is the confidence coefficient (95\%), $n$ is the amount of data.

The statistical parameters of flares as a function of area class are shown in Figure 5.

The results indicate that as importance and area class increase, so do the statistical parameters of flares. The mean duration of the flash phase increases from 4.6 to 16 min, modal times shift from 1 to 6 min; median ones, from 3 to $10 \mathrm{~min}$. Time intervals for $90 \%$ of flares also increase. A similar trend is observed for flare brightness characteristics (Tables 2 and 3), although it is not clearly defined. The conclusion drawn by Borovik, Zhdanov [2017] also finds its confirmation: with increasing area class, the number of flares is redistributed toward increasing brightness class (see Table 2).

Table 2

Statistical parameters of solar flares as a function of importance and area class

\begin{tabular}{|l|r|c|c|r|c|}
\hline & \multicolumn{1}{|c|}{$N$} & $\bar{t} \pm \alpha$ & $M o$ & $M e$ & $\Delta t$ \\
\hline SF & 55065 & $4.6 \pm 0.1$ & 1 & 3 & $1-10$ \\
\hline SN & 18101 & $5.4 \pm 0.1$ & 2 & 3 & $1-12$ \\
\hline SB & 3819 & $5.9 \pm 0.3$ & 2 & 3 & $1-13$ \\
\hline 1F & 1919 & $10.5 \pm 0.6$ & 4 & 6 & $1-25$ \\
\hline 1N & 2847 & $10.6 \pm 0.5$ & 3 & 6 & $1-24$ \\
\hline 1B & 1569 & $10.8 \pm 0.7$ & 3 & 6 & $1-23$ \\
\hline$(2-4) \mathrm{F}$ & 167 & $14.5 \pm 2.6$ & 4 & 10 & $1-31$ \\
\hline$(2-4) \mathrm{N}$ & 450 & $16.0 \pm 1.6$ & 4 & 10 & $1-34$ \\
\hline$(2-4) \mathrm{B}$ & 664 & $15.5 \pm 1.3$ & 6 & 10 & $1-34$ \\
\hline S & 76985 & $4.9 \pm 0.1$ & 1 & 3 & $1-11$ \\
\hline 1 & 1281 & $10.6 \pm 0.3$ & 4 & 6 & $1-24$ \\
\hline $2-4$ & 1303 & $15.6 \pm 0.9$ & 4 & 10 & $1-34$ \\
\hline$\sum$ & 84601 & $5.5 \pm 0.1$ & 1 & 3 & $1-12$ \\
\hline
\end{tabular}


Statistical parameters of solar flares as a function of brightness class

Table 3

\begin{tabular}{|c|c|c|c|c|c|}
\hline Brightness & $N$ & $\bar{t} \pm \alpha$ & $M o$ & $M e$ & $\Delta t$ \\
\hline F & 69306 & $4.8 \pm 0.1$ & 1 & 3 & $1-11$ \\
\hline N & 25907 & $6.3 \pm 0.1$ & 2 & 4 & $1-14$ \\
\hline B & 7098 & $8.0 \pm 0.3$ & 2 & 4 & $1-19$ \\
\hline
\end{tabular}

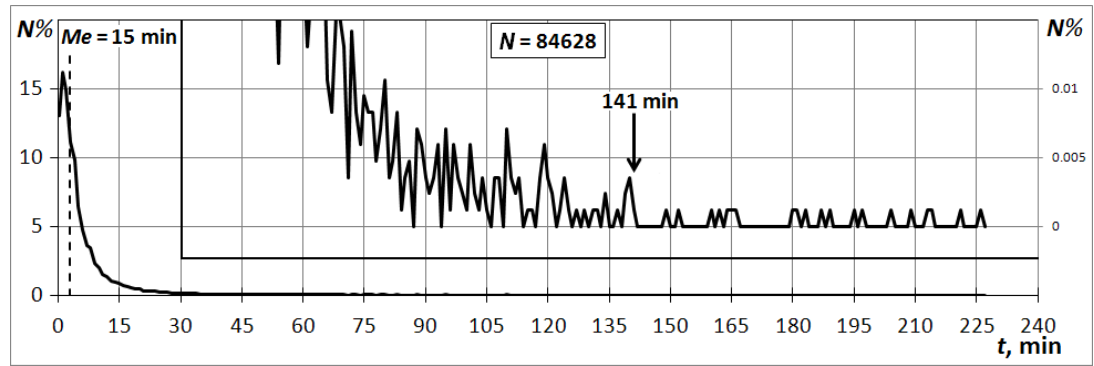

Figure 1. Distribution of solar flares by brightness rise time
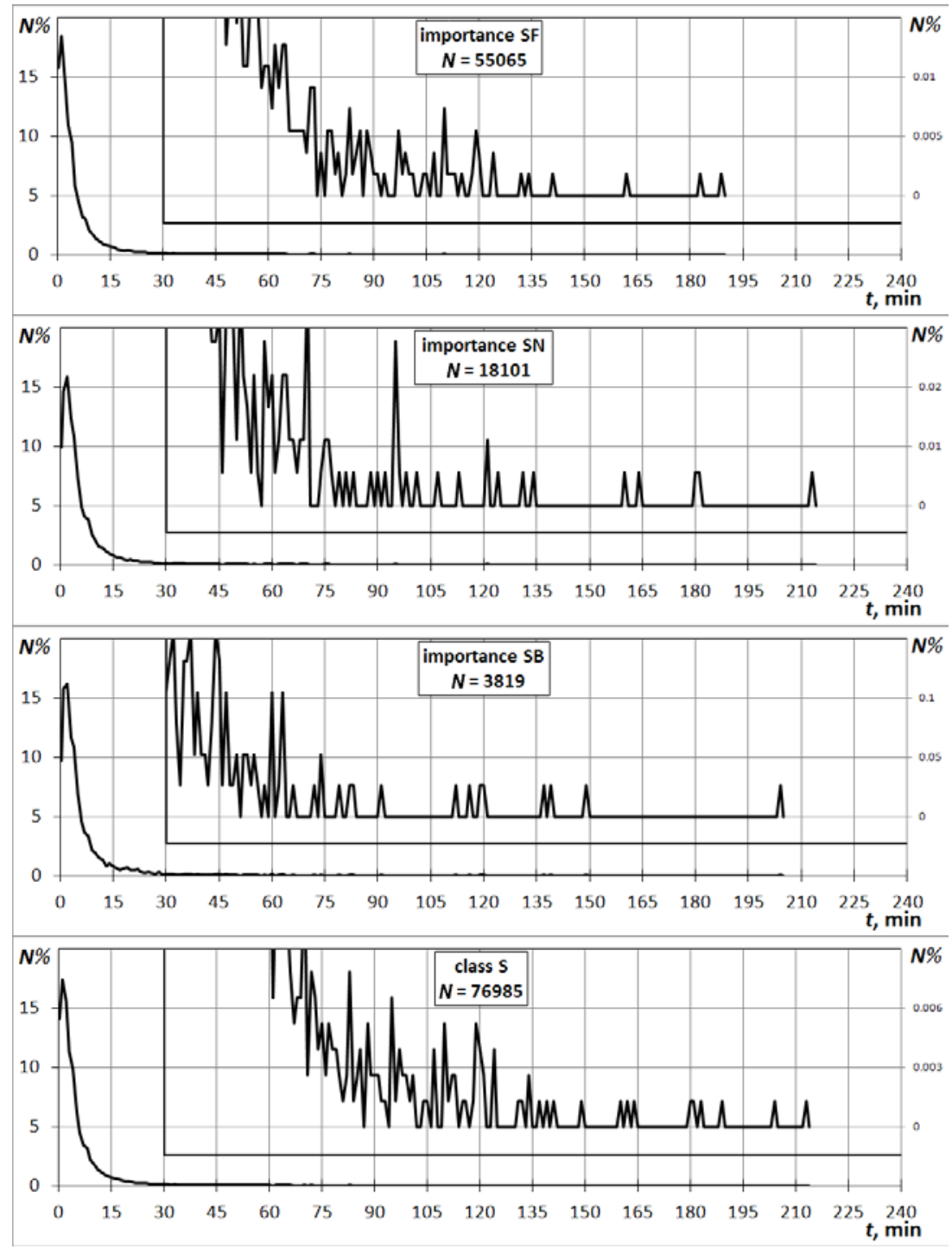

Figure2. Brightness rise time distribution of for class S flares 

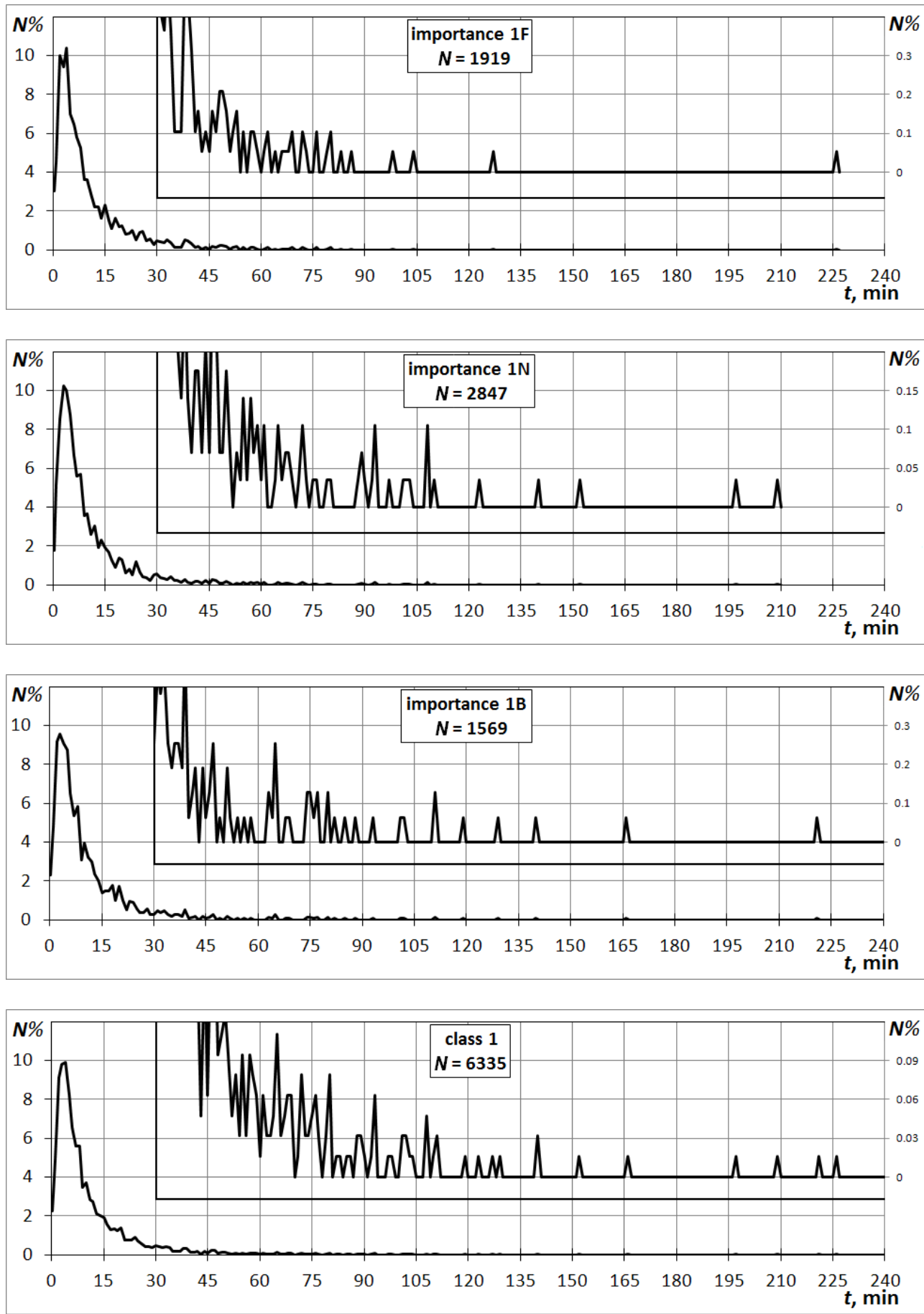

Figure 3. Brightness rise time distribution for class 1 flares 

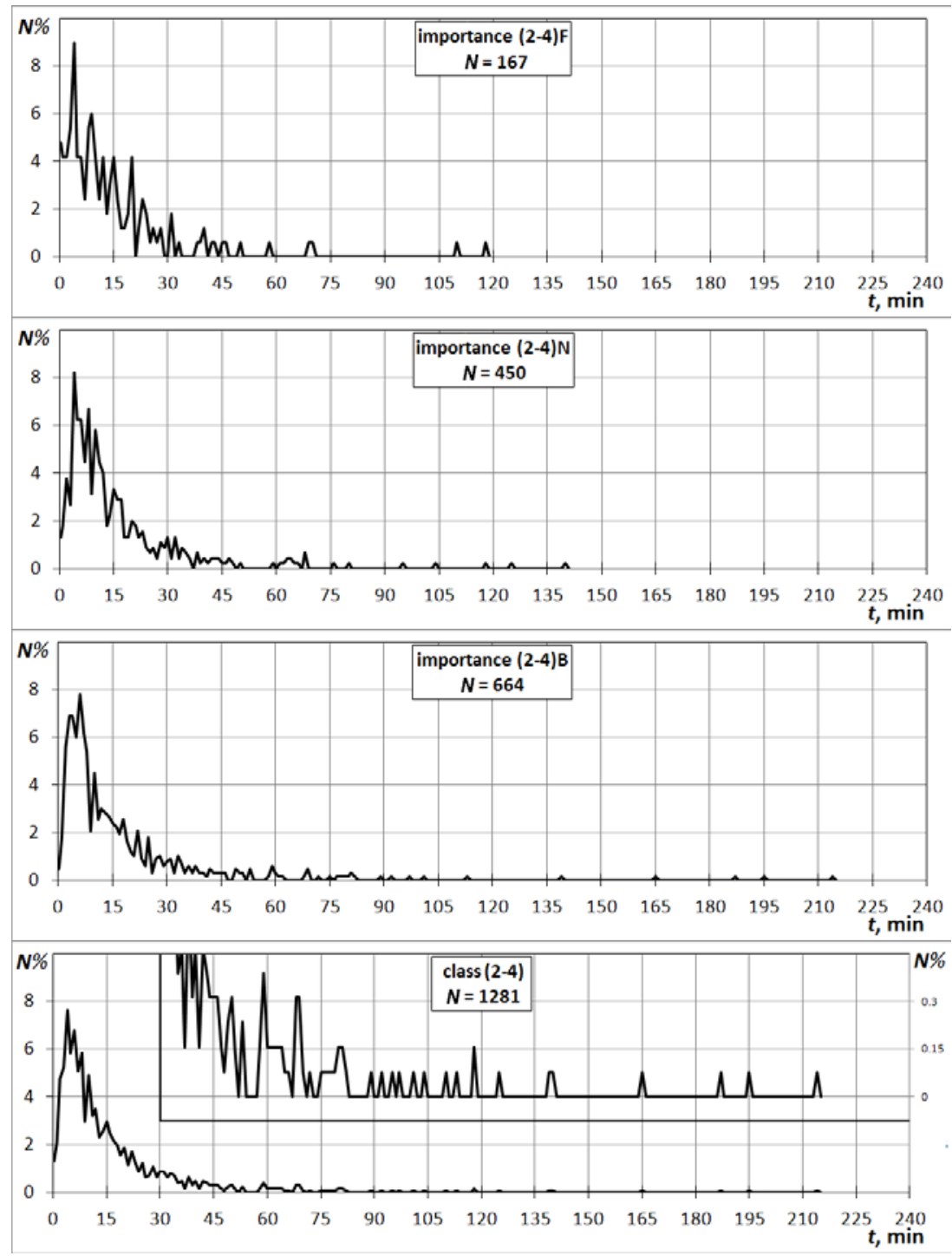

Figure 4. Brightness rise time distribution for class 2-4 flares

\section{BRIGHTNESS RISE TIME AS DERIVED FROM HOLL, LEAR, RAMY DATA}

To confirm the results, we conduct a similar analysis of data from HOLL (Holloman Solar Observatory, Air Force Base, New Mexico, USA), LEAR (Learmonth Solar Observatory, Australia), and RAMY (Ramey Solar Observatory, Puerto Rico).

The number of flares is shown in Table 4.

According to data from the three observatories, rise times for low-power flares differ slightly. For flares of higher area classes, they diverge within the range smaller than $\bar{\sigma}$ (Figure 6, $a$ ).

For flare importance (Figure 6, b) due to insufficiently high statistical significance of the data (especially for large flares) the differences are larger. Nevertheless, the rise time increase with increasing importance and area class is quite well defined.

Summarizing the above results, we can draw the following conclusions:

- Using extensive statistical material, we have confirmed the tendency of the brightness rise time to in- crease with increasing importance and area class of flares.

- We have obtained reliable values of the mean duration of the flash phase for different importance and area classes of solar flares (Tables 2 and 5, Column III).

Note that the smaller amount of data we utilize for the statistical analysis as compared to that in [Temmer et al., 2001] results from the use of a more rigid approach to the selection of solar flares.

\section{DURATION OF FLASH PHASE FOR FLARES OF INDIVIDUAL TYPES}

While the tendency for an increase in the rise time with area class is fairly pronounced, there is, in fact, virtually no direct functional connection with the area of flares. The correlation coefficient is less than 0.2 . Low-power flares exhibit especially significant scattering of data. Figure 7, a demonstrates a fairly strong mutual overlap of distributions such that $99 \%$ of lowpower flares and $95 \%$ of class 1 flares fall into the time interval for $90 \%$ of high-power flares $(\Delta t=1-34$ min) (Figure $7, b$ ). 


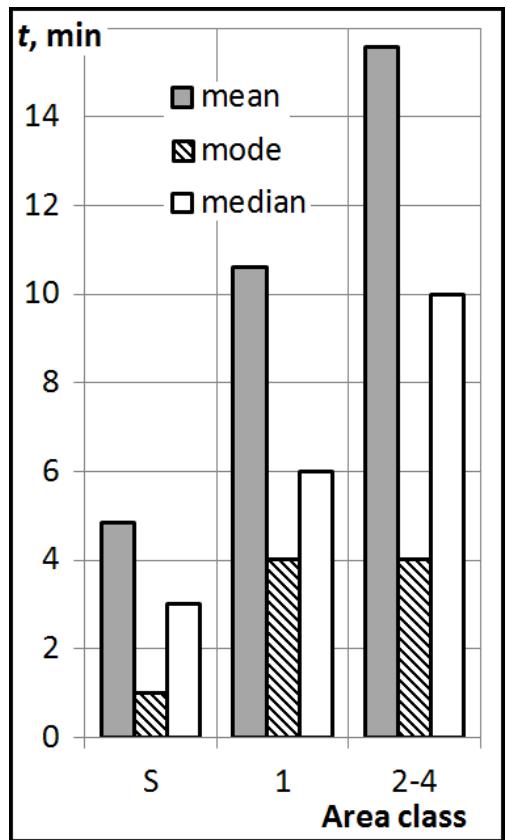

Figure 5. Statistical parameters of solar flares as a function of increasing area class

Table 4

The number of flares of different importance and area classes according to HOLL, LEAR, and RAMY data

\begin{tabular}{|l|r|r|r|r|}
\hline & \multicolumn{1}{|c|}{$\begin{array}{c}\text { all } \\
\text { stations }\end{array}$} & HOLL & LEAR & RAMY \\
\hline SF & 55065 & 14223 & 11815 & 8347 \\
\hline SN & 18101 & 2569 & 1639 & 1970 \\
\hline SB & 3819 & 973 & 341 & 840 \\
\hline $1 \mathrm{~F}$ & 1919 & 326 & 454 & 175 \\
\hline $1 \mathrm{~N}$ & 2847 & 419 & 447 & 199 \\
\hline $1 \mathrm{~B}$ & 1569 & 368 & 251 & 201 \\
\hline$(2-4) \mathrm{F}$ & 167 & 27 & 35 & 9 \\
\hline$(2-4) \mathrm{N}$ & 450 & 60 & 78 & 29 \\
\hline$(2-4) \mathrm{B}$ & 664 & 164 & 155 & 86 \\
\hline $\mathrm{S}$ & 76985 & 17765 & 13795 & 11157 \\
\hline 1 & 6335 & 1113 & 1152 & 575 \\
\hline $2-4$ & 1281 & 251 & 268 & 124 \\
\hline$\sum$ & 84601 & 19129 & 15215 & 11856 \\
\hline
\end{tabular}

To determine the reason for the tendency of $\bar{t}$ to increase, we statistically analyze the brightness rise time for flares of individual types. Stations report on some features of flares in the form of remarks. The system of remarks introduced by the international solar patrol generally characterizes the type of a flare, its attendant events, generating area, size of the active region, etc. (a total of 26). The most important ones are discussed below.

- D - Brilliant point;

- E - Two or more brilliant points;

- $\mathrm{G}$ - No visible spots nearby (spotless flares);

- $\mathrm{H}$ - Flare accompanied by high-speed dark filament;

- K - Several intensity maxima;

- L - Existing filaments suddenly active;

- $\mathrm{M}$ - White-light flare;

- $\mathrm{R}$ - Asymmetry in $\mathrm{H} \alpha$ line suggests high-speed mass ejection;
- S - Brightness followed filament disappearance in the same position;

- U - Two bright branches, parallel or converging (two-ribbon flare);

- V - Considerable expansion for about 1 min that often includes a significant intensity increase;

- W - Great area increase after time of maximum brightness;

- Z - Major sunspot umbra covered by flare.

Stations generally report on one or two features and very rarely on three-five. The frequency of their occurrence among flares of different area classes is shown in Figure 8. To analyze the frequency characteristics, we use only longitude limitations and exclude visual data.

Referring to the histogram, the above features can be found in almost all flares, regardless of their area class.

This suggests that low-power flares in terms of features of development do not differ from large flares. They, as well as powerful flares, occur with filament activation and disappearance (L, S, H), cover sunspot umbra in active regions (Z), may have an explosive phase $(\mathrm{V})$ and several intensity maxima (K). Among them are two-ribbon (U) and spotless $(\mathrm{G})$ flares. Even very rarely observed white-light flares (M) accompanied by powerful hard $\mathrm{X}$-rays and microwave radiation may have very low optical importance in the $\mathrm{H} \alpha$ line.

Unfortunately, because stations often do not report on features of flare development, we managed to analyze only those types of flares whose statistical significance is great enough, i.e. V, K, U, G, H, D, E flares.

The statistical parameters obtained for these flares are listed in Table 6. As for all flares, we do not consider data acquired visually and under poor observation conditions, as well as class $\mathrm{S}$ and 1 flares with heliolongitudes more than $65^{\circ} \mathrm{E}$ and $65^{\circ} \mathrm{W}$.

The data in Table 6 and Figure 9 show that explosive type flares (V) and flares with one brilliant point (D) have the shortest flash phases. Two-ribbon flares and $\mathrm{K}$ flares with several intensity maxima have the longest ones.

Spatial and temporal features of frequency characteristics of flares suggest that explosive type flares (V) often occur in places of filament disappearance (S). In many cases, there is one brilliant point (D) in the flare region. Unlike $\mathrm{V}$ flares, $\mathrm{K}$ flares whose light curves display several intensity maxima have a wider range of features. Among them is a higher percentage of tworibbon flares (U), flares with several brilliant points in a flare region (E), flares covering umbrae of large sunspots (Z). These features can partially explain the fact that the mean rise time of $\mathrm{V}$ flares is 2.8-2.1 times shorter than that of $\mathrm{K}$ flares. Also, in V flares, distribution medians are 2.0-2.4 times smaller and time intervals are 3.3-1.7 times shorter for $90 \%$ of flares.

The rise time seems to be connected with the number of brilliant points in the flare region $(D, E)$. The mean rise time in $\mathrm{D}$ flares is shorter than that in $\mathrm{E}$ flares. A distinctive feature of $\mathrm{D}$ flares is that they show their explosive nature (V) more often than E flares. Among them, $\mathrm{K}$ and $\mathrm{U}$ flares are rarer. 
Table 5

Statistical parameters of solar flares as a function of area class

\begin{tabular}{|c|r|r|r|r|r|r|r|r|r|r|c|c|}
\hline & \multicolumn{3}{|c|}{ Number } & \multicolumn{3}{c|}{ Mean } & \multicolumn{3}{c|}{ Mode } & \multicolumn{3}{c|}{ Median } \\
\cline { 2 - 14 } & \multicolumn{1}{|c|}{ I } & \multicolumn{1}{|c|}{ II } & \multicolumn{1}{c|}{ III } & \multicolumn{1}{c|}{ I } & II & III & I & II & III & I & II & III \\
\hline S & 2660 & 85649 & 76985 & 5.7 & - & 4.9 & $<2$ & - & 1 & - & 3 & 3 \\
\hline 1 & 211 & 9176 & 6335 & 10.1 & - & 10.6 & 5 & - & 4 & - & 5 & 6 \\
\hline 2 & 88 & 1014 & 1156 & 15.5 & - & 15.1 & 4 & - & 4 & - & - & 10 \\
\hline 3 & - & 101 & 115 & - & - & 21.2 & - & - & 6 & - & - & 17 \\
\hline $2-4$ & - & 1120 & 1281 & - & - & 15.6 & - & - & 4 & - & 8 & 10 \\
\hline$\sum$ & 2959 & 95945 & 84601 & - & 5.1 & 5.5 & - & 1 & 1 & - & 3 & 3 \\
\hline
\end{tabular}

Note: Column I shows the results obtained by Smith G., Smith E. [1966]; Column II, by Temmer et al. [2001]; Column III presents our results.

Table 6

Statistical parameters of solar flares of various types as a function of area class

\begin{tabular}{|c|c|c|c|c|c|c|c|c|c|c|c|c|c|c|c|}
\hline & \multicolumn{5}{|c|}{ class S } & \multicolumn{5}{|c|}{ class 1} & \multicolumn{5}{|c|}{ classes 2-4 } \\
\hline type & $N$ & $\bar{t} \pm \alpha$ & Mo & $M e$ & $\Delta t$ & $N$ & $\bar{t} \pm \alpha$ & Mo & $M e$ & $\Delta t$ & $N$ & $\bar{t} \pm \alpha$ & Mo & $M e$ & $\Delta t$ \\
\hline $\mathrm{V}$ & 2761 & $3.9 \pm 0.2$ & 2 & 3 & $1-8$ & 270 & $8.0 \pm 1.2$ & 3 & 5 & $1-18$ & 50 & $12.0 \pm 3.1$ & 4 & 8 & $1-31$ \\
\hline $\mathrm{D}$ & 3290 & $4.0 \pm 0.2$ & 2 & 3 & $1-8$ & 218 & $7.5 \pm 1.3$ & 2 & 4 & $1-19$ & 31 & $13.6 \pm 6.1$ & 3 & 7 & $1-28$ \\
\hline $\mathrm{H}$ & 2355 & $5.1 \pm 0.3$ & 1 & 3 & $1-11$ & 434 & $9.4 \pm 1.1$ & 3 & 6 & $1-20$ & 128 & $15.6 \pm 3.4$ & 4 & 8 & $1-38$ \\
\hline G & 484 & $5.8 \pm 0.5$ & 3 & 4 & $1-12$ & 68 & $9.2 \pm 2.2$ & 4 & 5 & $1-22$ & 6 & $15.3 \pm 5.8$ & 7 & 12 & $1-23$ \\
\hline $\mathrm{E}$ & 6858 & $6.0 \pm 0.2$ & 2 & 4 & $1-12$ & 1501 & $9.7 \pm 0.6$ & 2 & 6 & $1-21$ & 233 & $14.4 \pm 2.0$ & 4 & 10 & $1-31$ \\
\hline $\mathrm{U}$ & 570 & $7.6 \pm 0.8$ & 2 & 4 & $1-17$ & 429 & $13.2 \pm 1.5$ & 2 & 8 & $1-28$ & 229 & $19.7 \pm 2.5$ & 5 & 15 & $1-40$ \\
\hline $\mathrm{K}$ & 1027 & $10.9 \pm 0.8$ & 2 & 6 & $1-26$ & 309 & $19.5 \pm 2.5$ & 3 & 11 & $1-46$ & 97 & $25.0 \pm 4.7$ & 6 & 19 & $1-51$ \\
\hline & 76985 & $4.9 \pm 0.1$ & 1 & 3 & $1-11$ & 6335 & $10.6 \pm 0.3$ & 4 & 6 & $1-24$ & 1281 & $15.6 \pm 0.9$ & 4 & 10 & $1-34$ \\
\hline
\end{tabular}

Note: The last row lists statistical parameters for all flares.

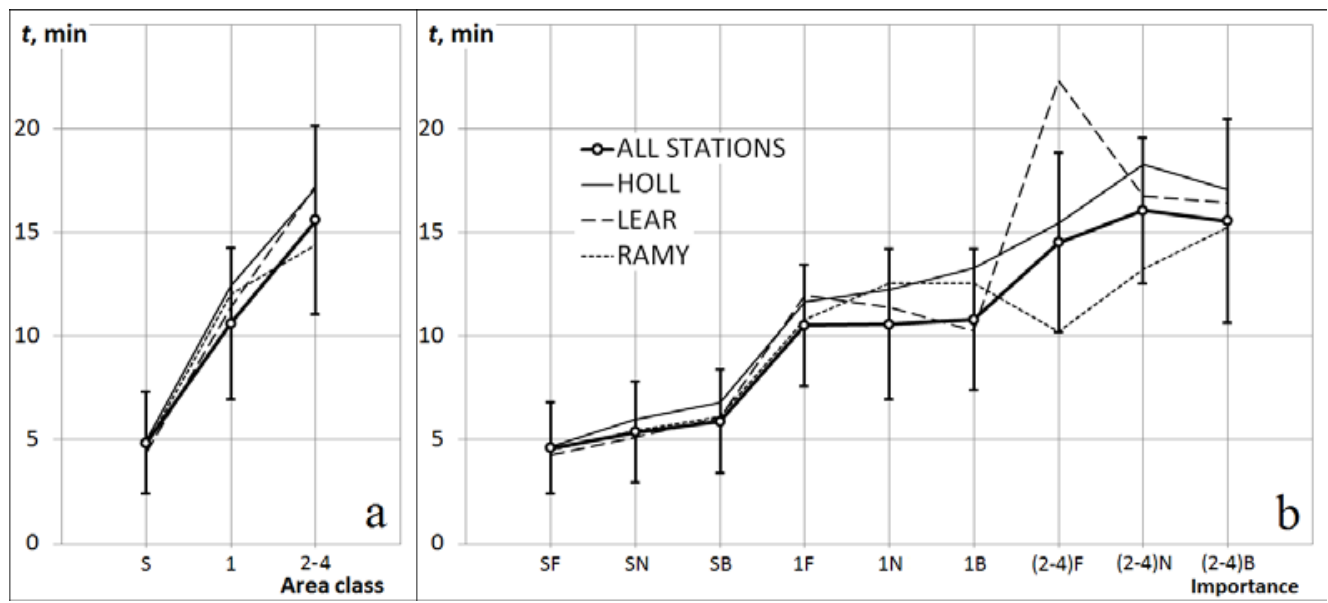

Figure 6. Variation in the mean brightness rise time with increasing area class (a) and importance $(b)$ according to HOLL, LEAR, and RAMY data. Vertical lines indicate $\bar{\sigma}$ scattering intervals

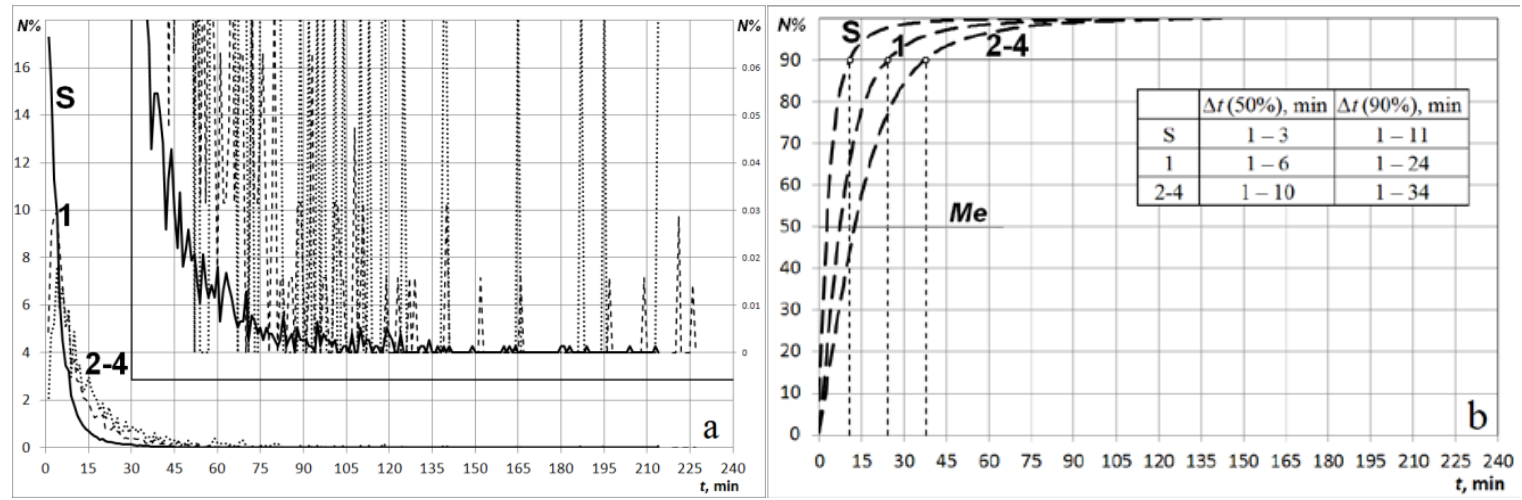

Figure 7. Distribution (a) of class S, 1 and 2-4 flares by the brightness rise time (solid line shows class S flares; dashed line, class 1 flares; dotted line, class 2-4 flares). The distribution's tails are scaled up (right axis); curves of cumulative frequencies of class S, 1, and 2-4 flares $(b)$. Table gives time intervals for 50 and $90 \%$ of flares 
The rise time of $U$ flares far exceeds the average time for all flares. They most often occur in active region, but can also be observed outside sunspots (G), are accompanied by filament activation (L) and high-speed dark filaments $(\mathrm{H})$. A high percentage of two-ribbon flares are $\mathrm{Z}$ flares. U flares may have several intensity maxima (K) and generally have several brilliant points in the flare region. As has been found in [Švestka et al., 1982], the brightest regions of flare ribbons are associated with footpoints of X-ray loops.

Among flares of the types considered, the parameters of $\mathrm{H}$ flares, followed by a high-speed dark filament, occupy a middle position, but are closer to the parameters of all flares. Their range of features is fairly wide. Such flares occur both in active regions with sunspots and outside sunspots (G), and can be seen among $\mathrm{D}$ and E flares. Among them are U, L, and K flares.

They most often appear in places of filament disappearance (Figure 10, a). In the Figure, Column V indicates the percentage of flares without additional features reported, as does Column U in Figure 10, $b$.

Unlike $\mathrm{V}$ flares, two-ribbon flares generally have two or more brilliant points. During their development, they often exhibit several intensity increases. This suggests

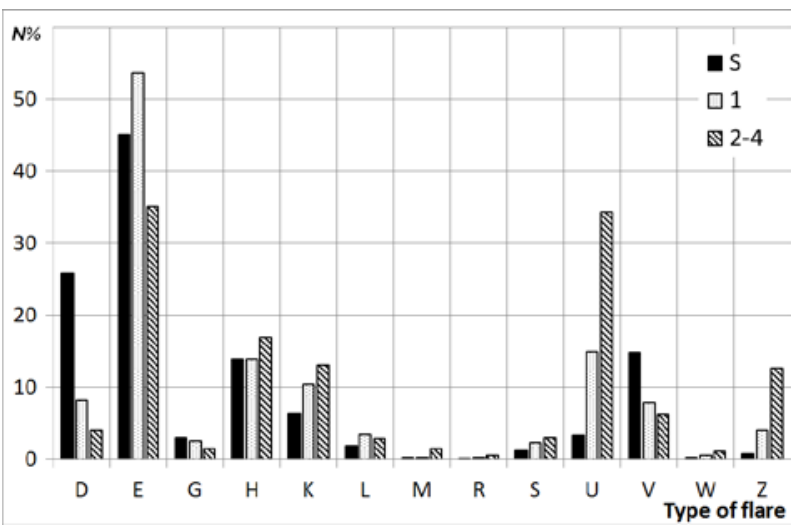

Figure 8. Frequency of occurrence of class S, 1 and 2-4 flares of different types. The number of flares is 20025,3701 , and 902 respectively that the physical nature of $\mathrm{V}$ flares differs from the nature of two-ribbon flares whose mechanism of occurrence is described by the CSHKP model.

They are most likely to be related to the so-called Hyder flares, which, according to Hyder's gravity model, may arise from the fall (return) of the eruptive prominence from the corona to the chromosphere [Hyder, 1967].

The analysis allows the following conclusions to be drawn:

- The mean brightness rise time comprises flash phases of flares of different types, the brightness rise times of which differ significantly.

- We can assume that, since the percentage of flares of different types and classes varies with solar cycle phase, the mean brightness rise time varies as well: decreases by minimum and increases by maximum solar activity.

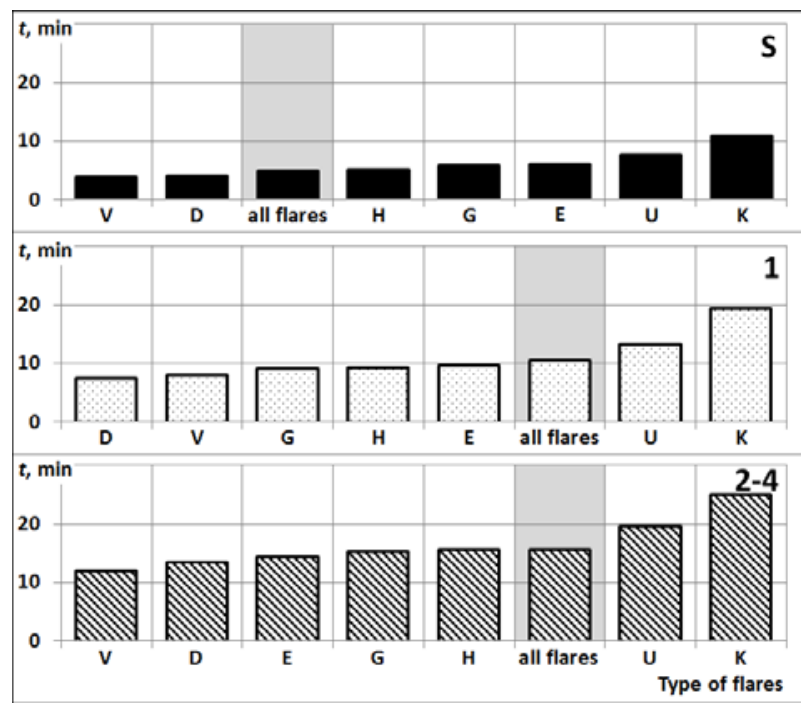

Figure 9. Distributions of mean rise time by area classes for flares of individual types. Histograms are plotted in ascending order of $\bar{t}$

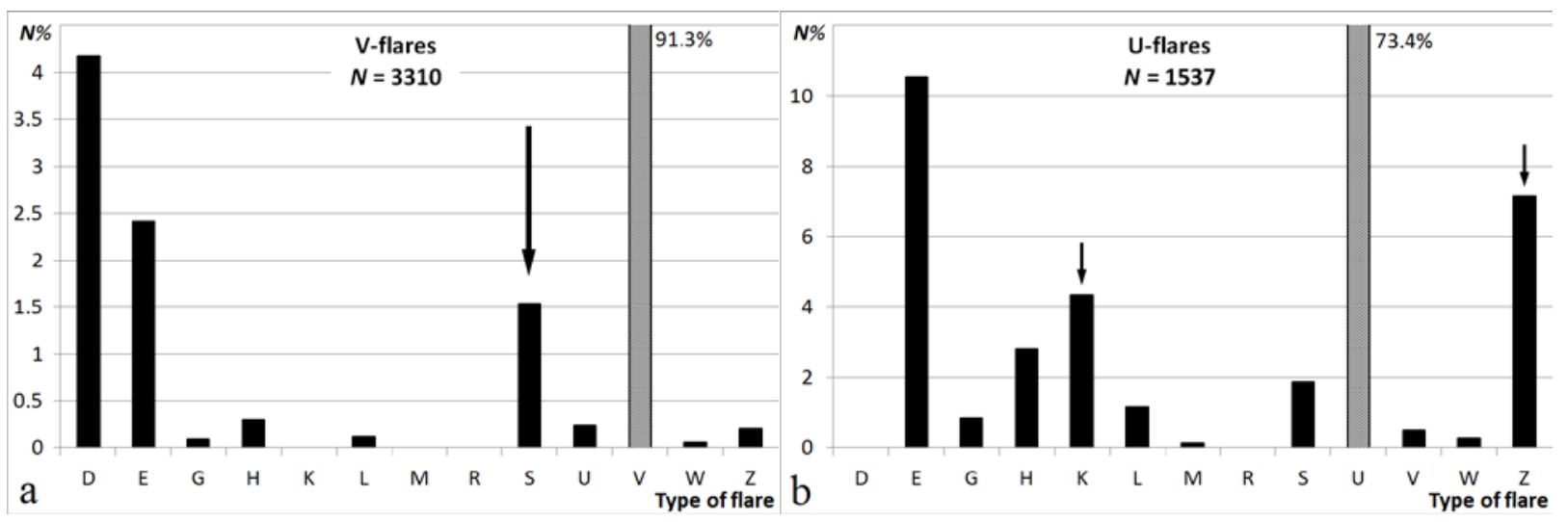

Figure 10. Features of development of explosive type flares $(a)$ and two-ribbon flares $(b)$ 


\section{ULTRA-LONG FLASH PHASES OF SOLAR FLARES}

From different sources it follows that the flash phase of solar flares usually lasts no more than one hour. There are, however, cases when it lasts much longer (more than $60 \mathrm{~min}$ ). Over the period of interest, 572 such flares (Table 7) have been detected.

Table 7

Statistical parameters of ultra-long solar flares as a function of area class

\begin{tabular}{|l|r|c|c|c|c|c|}
\hline & \multicolumn{1}{|c|}{$N$} & $N, \%$ & $\bar{t} \pm \alpha$ & $M e$ & $\Delta t$ & $L$ \\
\hline $\mathrm{S}$ & 326 & 57.0 & $87.5 \pm 4.1$ & 74 & $1-121$ & 346 \\
\hline 1 & 177 & 30.9 & $91.4 \pm 6.5$ & 77 & $1-140$ & 349 \\
\hline $2-4$ & 69 & 12.1 & $92.4 \pm 7.9$ & 80 & $1-135$ & 214 \\
\hline$\sum$ & 572 & 100 & $89.3 \pm 3.2$ & 76 & $1-125$ & 349 \\
\hline
\end{tabular}

The obtained data show that ultra-long flash phases occur in flares of almost all classes. Most of them are low-power flares. The mean brightness rise time for each area class is roughly the same (90 min). For $90 \%$ of flares, it varies from 60 to $140 \mathrm{~min}$. The maximum brightness rise time ( $L$ distribution range) for class $\mathrm{S}$ flares is $346 \mathrm{~min}$; for class 1 flares, $349 \mathrm{~min}$; for large flares, $214 \mathrm{~min}$. About $80 \%$ of ultra-long flares feature a shorter main phase. The mean relative duration is 0.67. There is in fact no tendency of the rise time to increase with increasing class of area for such flares.

\section{CONCLUSIONS}

The results of the statistical analysis allow the following conclusions to be drawn:

1. We have confirmed the tendency of brightness rise time to increase with increasing area class [Smith G., Smith E., 1966; Temmer et al., 2001]. Using extensive statistical material, we have first obtained the most complete and reliable values of the mean duration of the flash phase for solar flares of different importance and area classes. We have shown that with a pronounced tendency there is no direct functional relationship between the rise time and area of flares.

2. We have established that the rise time depends on the type of flares and features of their development. We have first obtained values of the mean duration of the flash phase for flares of seven types. Explosive type flares and flares with one brilliant point in the flare region have the shortest flash phases. Two-ribbon flares and flares with several intensity maxima have the longest flash phases. Flares of the types considered, as well as all flares, have a tendency of the rise time to increase with increasing area class.

3. We have found that almost all two-ribbon flares have several brilliant points. A considerable part of medium- and high-power two-ribbon flares features several intensity maxima; a flare often covers a sunspot umbra. Many explosive type flares occur in places of filament disappearance. Most often these are flares with one brilliant point in the flare region. This may suggest that these flares differ in their physical nature.
4. We have identified 572 cases when the duration of flash phases was over $60 \mathrm{~min}$. About $80 \%$ of these flares feature a shorter main phase. The mean relative duration of the flash phase is 0.67 . There is no tendency of the rise time to increase with increasing area class.

5. We have established that low-power flares, which constitute the majority of solar flares (more than $90 \%$ ), in terms of features of development do not differ from powerful solar flares. They, as well as large flares, are accompanied by filament activation and disappearance, have an explosive phase and several intensity maxima. Among them are two-ribbon flares, flares covering sunspot umbra, white-light flares.

6. Basing on these facts, we can assume that the mean brightness rise times comprising flash phases of flares of individual types depend on solar cycle phase: decrease by minimum and increase by maximum solar activity.

The work was performed with budgetary funding of Basic Research program II.16 No. 1.6.

\section{REFERENCES}

Abramenko, S.I., Dubov E.E., Ogir M.B., Steshenko N.E., Shaposhnikov E.F., Tsap T.T. Photometry of solar flares. Izvestiya Krymskoi astrofizicheskoi observatorii [Bull. Crimean Astrophys. Observatory]. 1960, vol. 23, pp. 341-361. (In Russian).

Altas L. Spotless flare activity. Solar Phys. 1994, vol. 151, no. 1, pp. 169-176.

Altyntsev A.T., Banin V.G., Kuklin G.V., Tomozov V.M. Solnechnye vspyshki [Solar Flares]. Moscow, Nauka Publ., 1982. 246 p. (In Russian).

Barlas O., Altas L. The duration of spotless flares. Astrophys. Space Sci. 1992, vol. 197, no. 2, pp. 337-341. DOI: $10.1007 /$ BF00645745.

Borovik A.V., Myachin D.Yu. The spotless flare of March 16, 1981. I. Pre-flare activations of the chromospheric fine structure. Solar Phys. 2002, vol. 2005, no 1, pp. 105-116.

Borovik A.V., Myachin D.Yu. Structure and development of the spotless flare on March 16, 1981. Geomagnetism and Aeronomy. 2010, vol. 50, no. 8, pp. 937-949. DOI: 10.1134/ $\underline{\text { S0016793210080037. }}$

Borovik A.V., Zhdanov A.A. Statistical studies of lowpower solar flares. Distributions of flares by area, brightness and classes. Solar-Terr. Phys. 2017, vol. 3, no. 1, pp. 40-56. DOI: $\underline{10.12737 / \text { article_58f96fda7e3e76.83058648. }}$.

Borovik A.V., Myachin D.Yu., Tomozov V.M. Observation of spotless solar flares at Baikal Astrophysical Observatory of ISTP SB RAS and their interpretation. Izvestiya Irkutskogo gosudarstvennogo universiteta. Ser. Nauki o Zemle [The Bulletin of the Irkutsk State University. Ser. Earth Sciences]. 2014, vol. 7, no. 1, pp. 23-45. (In Russian).

Borovik A.V., Myachin D.Yu., Uralov A.M. Spotless flare model. Izvestiya Krymskoi astrofizicheskoi observatorii [Bull. Crimean Astrophys. Observatory]. 2016, vol. 112, no.1, pp. 38-46. (In Russian).

Chistyakov V.F. Spotless flares. Issledovaniya po geomagnetizmu, aeronomii i fizike Solntsa [Res. on Geomagnetism, Aeronomy and Solar Physics]. 1988, iss. 79, pp. 70-75. (In Russian).

Dodson H.W., Hedeman E.R., McMath R.R. Photometry of solar flares. Astrophys. J. Suppl. 1956, vol. 2, pp. 241-270.

Fletcher L., Dennis B.R., Hudson H.S., Krucker S., Phillips K., Veronig A., Battaglia M., Bone L., Caspi A., Chen Q., 
Gallagher P., Grigis P.T., Ji H., Liu W., Milligan R.O., Temmer M. An observational overview of solar flares. Space Sci. Rev. 2011, vol. 159, pp. 19-106. DOI: 10.1007/s11214-010-9701-8.

Giersch O. GONG Inter-site $\mathrm{H} \alpha$ flare comparison. $J$. Phys.: Conf. Ser. 2013, vol. 440, iss. 1, article id. 012006. DOI: 10.1088/1742-6596/440/1/012006.

Hirayama T. Theoretical model of flares and prominences. I: evaporating flare model. Solar Phys. 1974, vol. 34, no. 2, pp. 323-338.

Hyder C.L. A phenomenological model for disparitions brusques followed by flare-like chromospheric brightenings. Solar Phys. 1967, vol. 2, no. 3, pp. 267-284.

Kopetskaya F., Kopetsky M. Characteristics of large chromospheric flares from Frittseva, Kopetsky and Šhvestka Catalogue. Issledovaniya po geomagnetizmu, aeronomii $i$ fizike Solntsa [Res. on Geomagnetism, Aeronomy and Solar Physics]. 1971, iss. 2, pp. 117-130. (In Russian).

Kopp R.A., Pneuman G.W. Magnetic reconnection in the corona and the loop prominence phenomenon. Solar Phys. 1976, vol. 50, pp. 85-98.

Luo B. The flares of spotless regions. Proc. the Kunming Workshop "Solar Physics and Interplanetary Travelling Phenomena”, November 21-25, 1983, Kunming, China / Eds. de Jager C., Biao Chen. Beijing: Science Press, 1985, vol. 1, p. 718.

Potzi W., Veronig A., Riegler G., Amerstorfer U., Pock TH., Temmer M., Polanec W., Baumgartne D.J. Real-time flare detection in groundbased $\mathrm{H \alpha}$ imaging at Kanzelhöhe Observatory. Solar Phys. 2014, vol. 290, no. 3, pp. 951-977.

Rossada V.M. Statistical analysis of 6600 flares for 1965 1966. Vestnik Kievskogo gosudarstvennogo universiteta [Bul- letin of Kiev State University. Ser. Astronomy]. 1977, no. 19, pp. 49-55. (In Russian).

Smith H., Smith E. Solnechnye vspyshki [Solar Flares] Moscow, Mir Publ., 1966, 426 p. (In Russian). English edition: Smith H.J., Smith E. Solar Flares. Macmillan, 1963. 322 p.

Sturrock P.A. Model of the high-energy phase of solar flares. Nature. 1966, vol. 211, pp. 695-697.

Švestka Z. Solar Flares. Dordrecht, Holland: D. Reidel, 1976. $415 \mathrm{p}$

Švestka Z., Dodson-Prince H.W., Martin S.F., Mohler O.C., Moore R.L., Nolte J.T., Petrasso R.D. Study of the postflare loops on 29 July 1973. Solar Phys. 1982, vol. 78, no. 2, pp. 271-285. DOI: $10.1007 / \mathrm{BF} 00151609$.

Temmer M., Veronig A., Hanslmeier A., Otruba W., Messerotti M. Statistical analysis of solar Ha flares. Astron. Astrophys. 2001, vol. 375, pp. 1049-1061.

Warwick C.S. Some characteristics of solar flares. Astrophys. J. 1954, vol. 120, pp. 237-244.

Ward F., Cornevall R.F., Hendle R. Solar flare observations from a pair of matched instruments. Solar Phys. 1973, vol. 31, no. 1, pp. 131-141.

Yatini C. Y. Characteristics of $\mathrm{H} \alpha$ Flare in the Solar Spotless Area. National Institute of Aeronautics and Space of Indonesia Majalah LAPAN. 2001, vol. 3, p. 53.

How to cite this article

Borovik A.V., Zhdanov A.A. Distribution of low-power solar flares by brightness rise time. Solar-Terrestrial Physics. 2018. vol. 4, iss. 3, pp. 3-12. DOI: $10.12737 /$ stp-43201801. 